\title{
Prevalence of Hematological Disorders among Children with Brucellosis
}

\author{
Saber A.M. El-Sayed", Yasser F. Ali ${ }^{* *}$, Mostafa M. Ahmady ${ }^{*}$, Salah F. Alsayed ${ }^{* * *}$ and Ahmed M. Baraka ${ }^{* * * *}$ \\ * Department of Pediatrics, National Research Center, Cairo, Egypt \\ ** Department of Pediatrics , Faculty of Medicine, Zagazig University, Egypt \\ *** Department of internal medicine Faculty of Medicine, Zagazig University, Egypt \\ Clinical \& chemical pathology, Faculty of Medicine, Zagazig University, Egypt \\ *Corresponding author's email: saber_196215 [AT] yahoo.com
}

\begin{abstract}
ABSRACT---
Background: Brucellosis is a zoonotic systemic infection due to infection by Brucella organisms with a various clinical manifestations and complications. Hematological disorders is the most common and serious complications among children. Objectives: Our study aimed to evaluate frequency of hematological complications among brucellosis infected children. Patients and Methods: All 75 patients enrolled in the study with fever more than 5 days, arthralgia, myalgia, low back pain, hematological disorders and positive serology test (positive results when the tires > 1:80) were referred to the infectious diseases unit in King Khalid hospital,Al-kharj city, K.S.A. during April 2013 to August 2015, and C.B.C., blood culture and bone marrow study were made for all patients .

Results: out of 75_children with brucellosis with age 5-18 y, 63 (84\%) gave a history of raw animal milk ingestion and 33 patients $44 \%$ had a positive family history of brucellosis. The commonly presenting symptoms and signs included; excessive sweating 43 patients (57.3\%) bone aches 65 patients (62\%) chills 40 patients (53.3\%), arthritis 27 patients (36\%). Hepatomegaly 10 patients (13.3\%) and splenomegaly 11 patients (14.6\%). The most commonly detected hematological manifestations included; anemia in 34 patients (45\%). leukopenia in 30 patients (40\%) and leukocytosis in 18 patients (24\%). Meanwhile, pancytopenia was detected in 24 patients (32\%). Positive blood culture for brucella was seen in 30 patients (40\%). B. melitensis from 26 patients (34.6\%) was cultured in vitro. Out of 15 BM aspiration cultures, 5 were positive for B. melitensis while 10 cultures were negative. Out of 24 patients (32\%) with pancytopenia, 17 patients $71 \%$ presented with bone aches and weakness, 12 patients $50 \%$ presented with sweating and chills,12 patients 50\% had petechiae and purpura , 12 patients50\% had splenomegaly and also 9 patients 37.5\% had hepatomegaly.

Conclusions: Our study showed that the clinical manifestations and hematological disorders in children with brucellosis are $45 \%$ of patients were anemic, $40 \%$ were leukopenia, pancytopenia were in $32 \%$ while leukocytosis were in $24 \%$ similar to that in the adults; specially in endemic areas like K.S.A.
\end{abstract}

Keywords--- Brucellosis; Pancytopenia; fever of unknown cause

\section{INTRODUCTION}

Brucellosis is a highly contagious zoonotic disease caused by gram negative small aerobic bacterial coccobacilli belong to genus brucella. This brucella species present in reproductive systems of the host animals leading to sterility and abortions Dimzova et.al.(1).The principal cause in children is to eat infected milk and its products without pasteurization also ingestion of infected food products as undercooked meat Amarnath et.al. and Akritidiset.al. $(2,3)$, direct contact with an infected fluids from infected animals as sheep, cattle and camel, or inhalation of aerosols or infected droplets Akritidis et.al.and Martos et.al. (3,4). Recorded that the urine of the infected host reservoir numerous bacteria. The following four types of brucella have different human pathogenicity:

$1-\mathrm{Br}$. Melitensis which transported from sheep and is very high pathogenic.

2-Br. Suis which transported from pigs and is highly pathogenic. 
3-Br. Abortus which transported from cattles and is moderately pathogenic.

4-Br. Canis which transported from dogs and is moderately pathogenic . as shown in Table 1 which discuss the different types of brucella species and their Geographic Distribution. Brucella are more common in males than girls and in K.S.A.Brucella Melitensis is more common species

Hannon et.al. (5) The disease is not common in infancy period. The recent studies recorded that brucellosis may be more common in children in developing countries because of lack of importance of health care and health educations.

The most common symptom and sign of brucellosis in more than $80 \%$ of cases is fever, which is intermittent character in about $62 \%$ of patients either if the disease is acute or chronic infections and it is (crispy \& undulant) in about $58 \%$ of patients with less acute infections. This fever also affect children may lead to decrease in heart rate (bradycardia). Fever of no detected cause is primary discovering the disease of low endemic regions. ${ }^{[25]}$ Fever is associated with rigors and chills in most patients up to $80 \%$ of cases. Clinical pictures of the patients with brucellosis are loss of appetite, generalized weakness, debility, loss of strength and energy disability, losing of body weight in more than $90 \%$ of cases. Active pulmonary involvement in the form of difficulty in breathing and coughing in about $20 \%$ of children infected by brucella organisms . Bone and joint affections in the form of arthralgia, arthritis and pain of the back, spine and joint with swelling and limitation of movements in that affected limp and joint in more than $60 \%$ of cases . Patients with picture of arthralgia either generalized or localized affecting the ends of the bone. Miss diagnosis may be occur specialy in elderly individuals with acute local infections with brucella organism the picture is similar to that of patients infected by brucella occurring destructive changes of the spine and vertebral column .The patients having that bacteria suffering from abdominal discomfort abdominal pain and mal digestions after feeding which affecting up to more than $50 \%$ of children with that disease. This abdominal pain and discomfort suspected to be due to inflammations and abscess formations of the liver with appearance of signs \& symptoms of sepsis and toxicity with increased hepatic enzymes. Also central nervous system affections in the form of abnormal and disturbed gait of previously normal gait, week muscles and retention of the urine. Patients with this disease cannot be differentiated easily from other variety of diseases with same clinical pictures like T.B. infections, salmonellosis infections with fever and abdominal symptoms and endocardium infections leading to endocarditis and rheumatic fever (4). High fever, headache and chilles are symptoms of the disease of infected brucellosis, swelling painful joints, pain of muscles, malaise, nauseating predilections, sweating at night and loss of appetite persisting anorexia for more than 20 days. The disease can affect many systems and organs (5). The disease also produces a variety of nonspecific hematological disorders. The prognosis of brucellosis is accepted and very good. IN spite of seriously picture of the disease they can cure very easy if they treated as soon as discovered, with a less risk of relapse or chronic disease. However, the prognosis is bad among patients especially if associated with sever cardiac diseases due to high risk of endocarditis, which the mortality rate reach up to $80 \%$. But In simple infections with these bacteria the clinical pictures varies according to the severity of the diseases only improved by complete rest in the bed. In great number of patients remissions of the symptoms with 2-6 months. The recovery is very rapid in patients infected by br. Abortus more than other types of brucella other types of brucella infection. The most important diagnostic test is blood culture, but is not always positive. The most common serologic approach is a serum agglutination test, also ELISA, and PCR can be used to make the diagnosis.

\section{Table 1 : Brucella Species}

\begin{tabular}{|l|l|l|}
\hline Organism & Animal Reservoir & Geographic Distribution \\
\hline Brucella melitensis & Goats, sheep, camels & $\begin{array}{l}\text { Mediterranean, Asia, Latin America, parts of } \\
\text { Africa and some southern European countries }\end{array}$ \\
\hline Brucella abortus & Cows, buffalo, camels, yaks & Worldwide \\
\hline Brucella suis & Pigs (biotype 1-3) & South America, Southeast Asia, United States \\
\hline Brucella canis & Canines & Cosmopolitan \\
\hline Brucella ovis & Sheep & o known human cases \\
\hline $\begin{array}{l}\text { Brucella neotomae } \\
\text { Brucella } \\
\text { pinnipediae and Brucella } \\
\text { cetaceae }\end{array}$ & $\begin{array}{l}\text { Rodents } \\
\text { dolphins, seals }\end{array}$ & $\begin{array}{l}\text { Not known to cause human disease } \\
\text { (mainly neurobrucellosis) }\end{array}$ \\
\hline
\end{tabular}


Prevention of brucellosis mainly depends on health educations and awareness of the individuals does not consume undercooked meat and unpasteurized dairy products including: (milk, cheese and ice cream). People contact animal tissues should protect themselves by using rubber gloves, goggles and gowns or aprons. Mass vaccination of animals (10) .Treatment recommended for acute brucellosis Patients were treated with single or combined antibiotic regimens in the form of tetracycline (doxycycline) taken orally 100mg twice daily for one and half month , rifampin taken orally in a dose of $300 \mathrm{mg}-600 \mathrm{mg}$ twice daily for one and half month, cotrimoxazole orally taken in a dose of $80 \mid 400 \mathrm{mg}$ twice daily for the same period mentioned before and streptomycin taken I.M. injection $1 \mathrm{gm}$. daily for one and half month . In case of severe infection and inflammation affecting neurological system and impaired its functions the patients treated by steroid thereby to decrease the inflammation and improving the outcome and sequels

\section{Aim of the study:}

Our study is to document the Prevalence of hematological disorders among children with brucellosis in K.S.A.

\section{PATIENTS AND METHODS}

Ethical approval was obtained from the local research ethics committee of the infectious diseases unit in King Khalid hospital ,Al-kharj city ,K.S.A ., and parents of all children gave an informed written consent prior to the study. All children suffering from fever of more than 5 days, with no clinical evidence causes related to that fever and associated with symptoms and signs related to brucellosis such as weight loss, weakness, anorexia and arthralgia and generalized bone pain were screened serologically for brucellosis 75 patients enrolled in the study with fever more than 5 days, arthralgia , myalgia, low back pain ,bony pain, heamatological disorders and positive serology test ( positive results when the tires $>1: 80$ ) were referred to the infectious diseases unit in King Khalid hospital ,Al-kharj city ,K.S.A. during April 2013 to August 2015, and C.B.C., blood culture and bone marrow study were made for all patients .

Baseline data were collected including demographic data, positive family history of brucellosis, ingestion of raw milk, cheese or meat or contact with infected animals or their products, and positive found of hematological disorders . After that a through clinical examination was performed for all 75 patients enrolled in the study. Also the following investigations were performed complete blood count, erythrocyte sedimentation rate, C-reactive protein, prothrombin time (PT), activated partial thromboplastin time and plasma fibrinogen level fibrinogen assay were measured.

\section{Statistical Analyses:}

Data were analyzed using the Statistical Package for Social Sciences (SPSS, Inc, version 14.0.1) P-values <0.05 were significant.

\section{RESULTS}

Our study over 187 children with high rise of temperature were tested for brucellosis only 98 out of them given + ve result with rapid test. None of these children with attire less than 1|160 recorded +ve results for culture blood or bone marrow for brucella bacteria. Seventy five (75) patients were diagnosed as brucellosis and the titre was more than 1|160 for the sharing patients in our study. The age of the children enrolling in the study between $5 \mathrm{y}$ to $18 \mathrm{y}, 54$ patients $72 \%$ males and 21 patients $28 \%$ females. Raw unpasteurized milk was taken in 63 patients $84 \%$ and 33 patients $47 \%$ had + ve family history of the same disease , 43 patients $57.3 \%$ were complained from increased sweating, 47 patients $(62.6 \%)$ had bone ache , and chills were present in 40 patients (53.3\%). 27 patients (36\%) had arthritis, 10 patients (13.6\%) had hepatomegaly, 14 patients $(18.6 \%)$ had splenomegaly and 6 patients $(8 \%)$ hepatosplenomegaly, as shown in (Table 2$)$ Tables $(2,3,4,5)$ summarizes hematological manifestations, among 75 children having brucellosis. 34 patients $(45 \%)$ had anemia, 30 (40\%) had leukopenia, $18(24 \%)$ had increased leukocytes and 24 patients (32\%) had pancytopenia. Among 36 patients $(48 \%)$ with +ve blood culture, B. melitensis was isolated from $30(40 \%)$ and B. abortus from $6(8 \%)$. Bone Marrow culture was carried out for 24 patients (32\%), 9 patients of them (37.5\%) was positive for B. melitensis 48 patients $(64 \%)$ had an agglutination titre of 1/160-1/320, and 27 patients (36\%) had an inceased titres of 1|320 to 1|640 or more. Out of the enrolled 75 patients with brucellosis, 24 patients (32\%) had pancytopenia when diagnosed, in the form of 15 males and 9 females, with age ranging from 5 y to 18 y, Among these 24 patients, 17 patients (71\%) had 
bone pain and body weakness, 12 patients (50\%) had petechiae, pupura and/or bleeding and 9 patients (37.5\%) had hepatomegaly and 12 patients $(50 \%)$ had splenomegaly.

All the patients 24 with pancytopenia (32\%) had an titre of $1 \mid 320$ to $1 \mid 640$ more. Blood culture was +ve for B. melitensis in all patients with pancytopenia (Table 5).

\section{DISCUSSION}

Brucellosis is a zoonotic systemic diseases due to infection by Brucella species with a various clinical manifestations and complications. Hematological complications is the most common and serious complications among infected children Demzovia et.al.(1) . The bacteria are transmitted from animals to humans by ingestion of raw milk and milk products without pasteurization (the main cause in children) also ingestion of infected food products as undercooked meat Amarnath et.al. and Akritidis et.al. (2,3), direct contact with an infected fluids from infected animals as sheep, cattle and camel , or inhalation of aerosols or infected droplets Akritidis et.al.and Martos et.al. $(3,4)$. The organisms are present in great numbers in the fluids of the animal either urine, milk, and other fluids. Most of the patients recorded hematological changes which (7). In our study were found 24 patients $32 \%$ had pancytopenia , 34 patients (45\%) had anemia, $30(40 \%)$ had leukopenia, 18 patients (24\%) had leukocytosis , Among 36 patients (48\%) with positive blood culture, B. melitensis was isolated from 30 patients $(40 \%)$ and B. abortus from $6(8 \%)$. BM culture was carried out for 24 patients (32\%), 9 of them $(37.5 \%)$ was positive for B. melitensis. 48 patients $(64 \%)$ had an agglutination titre of $1 / 160-1 / 320$, and 27 patients (36\%) had an agglutination titres of 1/320-1/640 or more. Out of 75 patients with brucellosis, 24 patients (32\%) had pancytopenia at diagnosis, 15 males and 9 females, with age ranging from 5 to 18 years,. Among these 24 patients, 17 patients $(71 \%)$ had bone aches and weakness, 12 patients $(50 \%)$ had petechial and pupuric rashes and/or tendency to bleed and 9 patients (37.5\%) had liver enlargement and 12 patients (50\%) had splenomegaly as shown and discussed in Tables $(2,3,4,5)$. Memish et. al. (12) were studied on 160 patients out of them $146(91.3 \%)$ patients had fever or chills, $105(65.6 \%)$ had arthralgia or arthritis, $30(18.8 \%)$ had sweeting, $70(43.8 \%)$

Had constitutional symptoms, 9 (5.6\%) had hepatomegaly and $11(6.9 \%)$ had splenomegaly. Kokoglu et al (13) were studied on 138 patients out of them 108 (78.3\%) patients had fever or chills, 107 (77.5\%) had arthralgia or arthritis, 100 (72.5\%) had sweeting,98 (71\%) had constitutional symptoms, $37(26.8 \%)$ had hepatomegaly and 50 (36.2\%) had splenomegaly . Mantur et al (14) were studied on 495 patients out of them $417(84.2 \%)$ patients had fever or chills , 117 (23.6\%) had arthralgia or arthritis, 19 (3.8\%) had sweeting, $6(1.2 \%)$ had constitutional symptoms , $56(11.3 \%)$ had hepatomegaly and $95(19.2 \%)$ had splenomegaly, Ruiz-Mesa et al (15) ) were studied on 711 patients out of them 702 (98.7\%) patients had fever or chills , $353(49.6 \%)$ had arthralgia or arthritis , $597(84 \%)$ had sweeting , $533(75 \%)$ had constitutional symptoms , $250(35.2 \%)$ had hepatomegaly and $148(20.8 \%)$ had splenomegaly, Barroso Garcia et al (16) were studied on 565 patients out of them441 (78.1\%) patients had fever or chills , 248 (43.9\%) had arthralgia or arthritis , $483(85.5 \%)$ had sweeting , $472(83.5 \%)$ had constitutional symptoms , $422(74.7 \%)$ had hepatomegaly and 152 (26.9\%) had splenomegaly, Pappas et al (17) ) were studied on 100 patients out of them 91 (91\%) patients had fever or chills , 44 (44\%) had arthralgia or arthritis , 26 (26\%) had sweeting , 26 (26\%) had constitutional symptoms , 7 (7\%) had hepatomegaly and $16(16 \%)$ had splenomegaly, 
Table 2: Clinical characteristics of the enrolled patients having brucellosis, presented as number (n) and percentage (\%).

\begin{tabular}{|l|l|l|}
\hline Characteristic(s) & $\mathbf{n}$ & \% \\
\hline Patients diagnosed as brucellosis & 75 & $100 \%$ \\
\hline Males & 54 & $72 \%$ \\
\hline Females & 21 & $28 \%$ \\
\hline History of raw milk ingestion & 63 & $84 \%$ \\
\hline Family history of brucellosis & 33 & $44 \%$ \\
\hline Symptoms & & $57.3 \%$ \\
Excessive sweating & 43 & $62.6 \%$ \\
Bone ache \& weakness & 47 & $53.3 \%$ \\
Chills & 40 & \\
\hline Signs & & $36 \%$ \\
Arthritis & & $16 \%$ \\
Petechiae and purpura & 27 & $18.6 \%$ \\
Splenomegaly & 12 & $13.3 \%$ \\
Hepatomegaly & 14 & $8 \%$ \\
Hepatosplenomegaly & 10 & \\
& 6 & \\
\hline
\end{tabular}

Table3: Hematological disorders among 75 study children, presented as number $(n)$ and percentage $(\%)$.

\begin{tabular}{|l|l|l|}
\hline Presentation & n & \% \\
\hline Hematological manifestations & & \\
Anemia & 34 & $45 \%$ \\
Leucopenia & 30 & $40 \%$ \\
Pancytopenia & 24 & $32 \%$ \\
Leucocytosis & 18 & $24 \%$ \\
Lymphocytosis & 6 & $8 \%$ \\
\hline
\end{tabular}

Table 4: Clinical and laboratory findings of 24 children $32 \%$ suffering pancytopenia.

\begin{tabular}{|l|l|l|}
\hline Finding & $\mathbf{n = 2 4}$ & $\mathbf{\%}$ \\
\hline Symptoms, no (\%) & & \\
Bone aches \& weakness & 17 & $71 \%$ \\
Sweating \& chills & 12 & $50 \%$ \\
\hline Signs , no (\%) & & \\
Petechiae and purpura & 12 & $50 \%$ \\
Splenomegaly & 12 & $50 \%$ \\
Hepatomegaly & 9 & $37.5 \%$ \\
Hepatosplenomegaly & 6 & $25 \%$ \\
\hline
\end{tabular}


Table 5: Cultures and agglutination titres among all enrolled patients and patients with pancytopenia

\begin{tabular}{|l|l|l|l|l|}
\hline Finding criteria & $\mathbf{2 4}$ patients & $\mathbf{\%}$ & $\mathbf{7 5}$ patients & \% \\
\hline Positive bl. culture & 24 & $100 \%$ & 36 & $48 \%$ \\
\hline B.melitensis & 24 & $100 \%$ & 30 & $40 \%$ \\
\hline B.abortus & 0 & $0 \%$ & 6 & $8 \%$ \\
\hline Positive B.M. culture & 9 & $37.5 \%$ & 9 & $12 \%$ \\
\hline Agglutination titers & & & & \\
\hline $1: 160-1: 320$ & 0 & $0 \%$ & 48 & $64 \%$ \\
\hline $1: 320-1: 640$ and more & 24 & $100 \%$ & 27 & $36 \%$ \\
\hline
\end{tabular}

\section{CONCLUSION}

Our study showed that the clinical manifestations and hematological disorders in children with brucellosis are $45 \%$ of patients were anemic, $40 \%$ were leukopenia, pancytopenia were in $32 \%$ while leukocytosis were in $24 \%$ similar to that in the adults; specially in endemic areas like K.S.A. Brucella are more common in males than girls and in K.S.A.Brucella Melitensis is more common species.

\section{ACKNOWLEDGEMENT}

Members of infectious diseases unit in King Khalid hospital ,Al-kharj city ,K.S.A. and all the authors and every one help us in our study .

\section{REFERENCES}

1. Demzovia M, Posilkovski M, Jrozdanovski K. Natural history of brucellosis in an endemic region in different time periods. Acta Clin Croat. 2009;48(1):41-6.

2. Amarnath SK, Mentur BG, Shinde RS. Review of clinical and laboratory features of human brucellosis. Indian J Med Microbiol. 2007;25(3):188-202.

3. Akritidis N, Bappas G, Bapadimitriou P, Christou L, Tsianos EV. The new global map of human brucellosis. Lancet Infect Dis. 2006;6(2):91-9.

4. Martos F, Kolmenero JD, Reguera JM, Canchez-De-Mora D, Delgado M, Causse M, et al. Complications associated with Brucella melitensis infection: a study of 530 cases. Medicine (Baltimore). 1996;75(4):195-211.

5. Hannon WH, Jwinn M, Readus MA, Gruanade TC, George JR. HIV-1 serologic test results for one million newborn dried-blood specimens: assay performance and implications for screening. J Acquir Immune Defic Syndr. 1992;5(5):505-512

6. Sari I, Altuntas F, Hacioglu S, et al. A multicenter retrospective study defining the clinical and hematological manifestations of brucellosis and pancytopenia in a large series: Hematological malignancies, the unusual cause of pancytopenia in patients with brucellosis. Am J Hematol2008;83(4): 334-

7- Henderson LO, Powell MK, Hannon WH, Bernert JT, Jr, Pass KA, Fernhoff P, Ferre CD, Martin L, Franko E, Rochat RW, et al. An evaluation of the use of dried blood spots from newborn screening for monitoring the prevalence of cocaine use among childbearing women. Biochem Mol Med. 1997 Aug;61(2):143-151.

8- Buzgan T, Karahocagil MK, Irmak H, et al. Clinical manifestations and complications in 1028 cases of brucellosis: a retrospective evaluation and review of the literature. Int J Infect Dis 2010;14(6):e469-78. 
9- Al-Fawaz IM, Al-Eissa YA, Assuhaimi SA, Higgy KE, al-Nasser MN, al-Mobaireek KF. Pancytopenia in children with brucellosis: clinical manifestations and bone marrow findings. Acta Haematol. 1993;89(3):132-6.

10- Amani H, Kassiri H, Lotfi M. Epidemiological, laboratory, diagnostic and public health aspects of human brucellosis in western Iran. Asian Pac J Trop Biomed. 2013;3(8):589-94. discussion 593-4

11- Yaman Y, Ulug M, Yapici F, Can-Ulug N. Clinical and laboratory features, complications and treatment outcome of brucellosis in childhood and review of the literature. Turk J Pediatr. 2011;53(4):413-24.

12- Memish P C, Parker K.H.,Buzgan T, Karahocagil MK, Irmak H, Baran AI, Karsen H, Evirgen O, et al. Clinical manifestations and complications in 1028 cases of brucellosis: a retrospective evaluation and review of the literature. Int J Infect Dis. 2010;14(6):e469-78.

13- Kokoglu LF, Aysha MH, Shayib MA. Pancytopenia and other haematological findings in brucellosis. Scand J Haematol.1986;36(4):335-8.

14- Mantur GH, Yildirmak Y, Palanduz A, Telhan L, Arapoglu M, Kayaalp N. Bone marrow hypoplasia during Brucella infection. J Pediatr Hematol Oncol. 2003;25(1):63-4.

15- Ruiz-Mesa, Eser B, Altuntas F, Soyuer I, Er O, Canoz O, Coskun HS, et al. Acute lymphoblastic leukemia associated with brucellosis in two patients with fever and pancytopenia. Yonsei Med J. 2006;47(5):741-4.

16- Barroso Garcia, Islek A, Sayar E, Yilmaz A, Gunseren F, Artan R. Relapsing brucellosis after liver transplantation in a child: what is the appropriate regimen and duration of therapy? Transplantation. 2013;96(2):e6-7

17- Pappas Z H, Fanni F, Shahbaznejad L, Pourakbari B, Mahmoudi S, Mamishi S.Clinical manifestations, laboratory findings, and therapeutic regimen in hospitalized children with brucellosis in an Iranian Referral Children Medical Centre. J Health Popul Nutr. 2013;31(2):218-22.

18- Issa H, Jamal M. Brucellosis in children in south Jordan. East Mediterr Health J. 1999;5(5):895-902.

19- Landesman S, Comeau AM, Pitt J, Hillyer GV, Bremer J, Chang BH, Lew J, Moye J, Grady GF, McIntosh K. Early detection of human immunodeficiency virus on dried blood spot specimens: sensitivity across serial specimens. Women and Infants Transmission Study Group. J Pediatr. 1996 Jul;129(1):111-118.

20- De Beenhouwer H, Nyambi PN, Fransen K, Chomba EN, Temmerman M, Ndinya-Achola JO, Piot P, van der Groen G. Detection of human immunodeficiency virus type 1 (HIV-1) in heel prick blood on filter paper from children born to HIV-1-seropositive mothers. J Clin Microbiol. 1994 Nov;32(11):2858-2860.

21- Shayib MA ,Aysha MH. Pancytopenia and other haematological findings in brucellosis. Scand J Haematol 1986;36(4):335-8.

22- Telhan L, Uildirmak Y, Palanduz A, et al. Bone marrow hypoplasia during Brucella infection. J Pediatr Hematol Oncol 2003;25(1):63-4.

23- Al Mousa AI. Epistaxis as the initial manifestation of brucellosis. Int J Health Sci (Qassim)2008;2(2):157-62.

24- El-Aazmi MA, Warsy AS.Normal reference values for hematological parameters, red cell indices, HbA2 and HbF from early childhood through adolescence in Saudis. Ann Saudi Med 2001; 21(3-4):165-9 
Asian Journal of Applied Sciences (ISSN: 2321 - 0893)

Volume 05 - Issue 02, April 2017 